Des données probantes pour façonner le changement social et comportemental en matière de planification familiale en Afrique de l'Ouest francophone

Breakthrough RESEARCH

Follow this and additional works at: https://knowledgecommons.popcouncil.org/departments_sbsr-rh How does access to this work benefit you? Let us know!

Recommended Citation

Breakthrough RESEARCH. 2020. "Des données probantes pour façonner le changement social et comportemental en matière de planification familiale en Afrique de l'Ouest francophone," Activity brief. Washington, DC: Population Council. 


\title{
Des données probantes pour façonner le changement social et comportemental en matière de planification familiale en Afrique de l'Ouest francophone
}

\begin{abstract}
La série d'activités de recherche de Breakthrough RESEARCH en Afrique de l'Ouest francophone vise à générer des données probantes pour déterminer à quel point différentes approches abordent les déterminants comportementaux et améliorent les résultats en planification familiale (PF) et en santé reproductive (SR). Cet exposé résume la création par Breakthrough RESEARCH d'un corpus de connaissances sur le changement social et comportemental (CSC).
\end{abstract}

L'Afrique de l'Ouest francophone présente les taux de fécondité les plus élevés au monde et un faible taux d'utilisation contraceptive. En 2011, neuf gouvernements de pays ouest-africains francophones ont constitué, avec des partenaires techniques et financiers, le Partenariat de Ouagadougou (PO) pour accélérer les progrès dans l'utilisation de services de PF dans la région. Depuis, les gouvernements des pays, les donateurs et les partenaires techniques œuvrent au renforcement des programmes PF/SR, essentiellement

INVESTISSEMENTS DE L'USAID DANS LE CSC EN AFRIQUE DE L'OUEST FRANCOPHONE

West Africa Breakthrough ACTION (WABA) est une initiative régionale financée par l'Agence américaine pour le développement international (USAID). Elle vise à renforcer la coordination et l'efficacité des interventions en matière de CSC dans quatre pays : le Burkina Faso, la Côte d'Ivoire, le Niger et le Togo. WABA œuvre en partenariat avec Amplify-FP, un autre projet financé par I'USAID, au renforcement de la prestation de servies dans ces quatre pays. Ces investissements servent de point d'entrée à Breakthrough RESEARCH pour étudier l'ampleur des programmes CSC dans la région, avant de déterminer et de documenter l'efficacité des approches dans ce domaine. par des investissements dans l'approvisionnement et la fourniture de produits et l'amélioration de la prestation de services. Les interventions en matière de CSC ont, toutefois, bénéficié d'investissements moindres.

Breakthrough RESEARCH, le projet de recherche phare de l'Agence américaine pour le développement international (USAID) en matière de CSC, vise à constituer un corpus de données probantes concernant l'efficacité des interventions dans ce domaine en Afrique de l'Ouest francophone, en particulier dans les pays de West Africa Breakthrough ACTION (WABA) : le Burkina Faso, la Côte d'Ivoire, le Niger et le Togo. Cet exposé résume le travail de Breakthrough RESEARCH avec les parties prenantes en PF/SR dans la région afin de mieux mesurer l'étendue du CSC et d'effectuer des évaluations ciblées des investissements de l'USAID dans ce domaine eu égard à la PF/SR.

\section{EXAMEN DU PAYSAGE DU CHANGEMENT COMPORTEMENTAL CHEZ LES PRESTATAIRES}

Les interventions sur le changement de comportement des prestataires (CCP) vont au-delà de la formation clinique et du soutien comme les aide-mémoire/supports techniques. Elles cherchent à influencer positivement le comportement des prestataires en vue d'améliorer la qualité des services, d'optimiser l'expérience des clients, d'accroître la demande de services et d'augmenter l'utilisation des produits ou l'adoption de comportements sains. Breakthrough RESEARCH a examiné l'étendue des données probantes disponibles

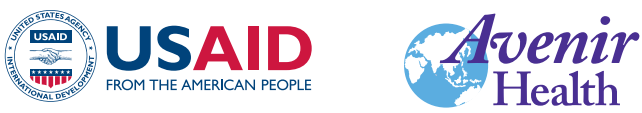

Institute for

Reproductive Health

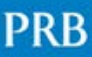




\section{INSTANTANE DES ACTIVITES}

Données probantes pour façonner le CSC en matière de PF en Afrique de l'Ouest francophone

\section{Consortium de Breakthrough RESEARCH}

Population Council (chef de file), Tulane University,

Avenir Health, ideas42, Population Reference Bureau

et Institute for Reproductive Health de Georgetown

University

\section{Partenaires collaborateurs}

Ministères de la Santé du Burkina Faso, de la Côte

d'Ivoire, du Niger et du Togo, Breakthrough ACTION,

Amplify-FP et institutions de recherche des pays

\section{Calendrier prévu}

Août 2019 - Août 2022

\section{Emplacement géographique}

Burkina Faso, Côte d'Ivoire, Niger et Togo.

\section{Contact}

Leanne Dougherty

Idougherty@popcouncil.org

\section{REVUE DOCUMENTAIRE DES MOYENS DE COMMUNICATION DE MASSE}

Breakthrough RESEARCH et son partenaire Tulane University ont effectué une revue documentaire des médias de masse et numériques et de la PF chez les jeunes en Afrique de l'Ouest francophone. Cet examen répertorie les données probantes concernant l'impact des médias de masse et numériques sur les résultats de la PF. Il indique aussi les domaines nécessitant une évaluation plus pointue.

Il inclut des documents publiés et issus de la littérature grise, ainsi que des expériences et des informations programmatiques émanant d'un certain nombre d'entretiens auprès d'interlocuteurs clés. Les enseignements tirés proviennent essentiellement de pays du PO mais aussi d'initiatives de communication menées dans toute l'Afrique et ailleurs.

Cet examen est destiné aux donateurs mondiaux et régionaux et aux concepteurs et agents de mise en œuvre de programmes PF, particulièrement en Afrique de l'Ouest francophone. Les recommandations portent sur la conception et l'exécution de stratégies de communication dans les médias de masse et numériques et sur l'augmentation de la base de données probantes concernant l'efficacité de ces programmes, en particulier sur les jeunes.

Pour de plus amples informations. Family Planning and Youth in West Africa : Mass media, digital media, and social and behavior change communication strategies, https://breakthroughactionandresearch.org/wp-content/ uploads/2019/09/Mass-Media-Literature-Review.pdf.

\section{SUIVI, ÉVALUATION ET ANALYSE DES COÛTS DE LA CAMPAGNE «MERCI MON HÉROS "}

Merci mon héros ( $\mathrm{MMH}$ ) est une campagne médiatique mise en œuvre par Breakthrough ACTION qui cible les jeunes et les adultes dans le but de promouvoir un environnement favorable à l'accès des jeunes à des services de PF/SR dans les quatre pays WABA. La campagne menée par des jeunes produit des vidéos via des plateformes de réseaux sociaux, notamment Facebook, Instagram, Twitter et YouTube, ainsi que par des canaux plus traditionnels comme la télévision, la radio, les événements de dépistage communautaires, les groupes de jeunes et autres. Breakthrough RESEARCH et son partenaire Tulane University évaluent la campagne MMH à travers trois activités séparées: (1) le suivi de l'écoute sociale (2) l'analyse de la performance qualitative et (3) l'analyse des coûts. Les résultats de ces activités façonneront la gestion adaptative de $\mathrm{MMH}$, ainsi que les programmes ultérieurs. Ces activités seront complétées par des enquêtes de suivi de routine qui seront effectuées par Breakthrough ACTION durant la campagne. 
Pour de plus amples informations. Merci Mon Héros, YouTube: https://www.youtube.com/

playlist?list=PLflIHdtzuE_E4sUlaMm7hwqNy8zOWykhN; Twitter:@MerciMonHeros,

Facebook: https://www.facebook.com/mercimonheros/, Instagram @MerciMonHeros; and https://

spark.adobe.com/page/oqbeLmPi1p4Ej/.

\section{Suivi des conversations du public de MMH à travers l'écoute sociale}

L'écoute sociale implique la collecte de données issues de réseaux sociaux et d'autres sources en ligne en utilisant un algorithme pour classer et coder le contenu en ligne. L'évaluation de $\mathrm{MMH}$ utilise cette technique pour suivre les communications en ligne et donner une idée des domaines d'intervention liés spécifiquement aux jeunes, à la sexualité et à la communication intergénérationnelle, ainsi qu'à de boucles de rétroaction rapides sur l'engagement dans la campagne et les réactions à des vidéos particulières.

Breakthrough ACTION se sert du rapport initial d'écoute sociale de Breakthrough RESEARCH pour déterminer certains thèmes, comme le rôle de la dimension de genre et la communication avec le partenaire, à incorporer dans les nouvelles vidéos de la campagne. Les rapports de suivi de l'écoute sociale ont également façonné la stratégie de $\mathrm{MMH}$. À titre d'exemple, le rapport sur l'écoute sociale a souligné que la campagne touchait essentiellement les jeunes et les jeunes adultes (âgés de 18 à 34 ans) mais pas les adultes plus âgés qui constituent un public crucial dès lors qu'il s'agit d'instiller une communication intergénérationnelle et de briser les tabous en parlant de la SR. En réponse, les animateurs de la jeunesse ont créé d'autres vidéos en ligne destinées spécifiquement aux adultes.

Produits à venir. Les conclusions des rapports sur l'écoute sociale seront résumées dans le rapport d'évaluation de MMH (décembre 2020). Un exposé séparé détaillant l'approche méthodologique sera diffusé pour informer les futurs efforts de suivi de la campagne sur les réseaux sociaux (Septembre 2020).

\section{Evaluation de la performance qualitative de la campagne $\mathrm{MMH}$}

L'évaluation de la performance qualitative de la campagne MMH de Breakthrough RESEARCH contribuera au corpus de données factuelles sur le CSC de deux manières : (1) en donnant une idée des conditions sous-jacentes favorisant la communication adultes/jeunes et (2) en déterminant les changements les plus significatifs dans les communautés exposées à la campagne médiatique au Niger et en Côte d'Ivoire. Les résultats seront communiqués à Breakthrough ACTION, aux partenaires locaux et aux parties prenantes gouvernementales pour examen et adaptation dans les futurs programmes d'engagement de la jeunesse.
Produits à venir. Les conclusions de l'évaluation qualitative seront résumées dans le rapport d'évaluation de MMH (Décembre 2020).

\section{Analyse des coûts de la campagne MMH} Avenir Health, partenaire de Breakthrough RESEARCH, effectue actuellement une analyse des coûts pour calculer les coûts financiers et économique de MMH en se servant des Directives sur l'établissement des coûts des interventions sur le CSC de Breakthrough RESEARCH.

Produits à venir. Produits à venir. Les conclusions d'Avenir Health seront résumées dans le rapport de rentabilité de MMH (Décembre 2020).

Pour de plus amples informations. Directives pour l'établissement des coûts des interventions en matière de CSC, https://breakthroughactionandresearch.org/wp-content/ uploads/2019/10/guidelines-for-costing-sbc-interventions. pdf; The Added Value of Costing Social \& Behavior Change Interventions, https://breakthroughactionandresearch. org/wp-content/uploads/2019/10/Costing-Brief.pdf.

\section{ENGAGEMENT DES HOMMES POUR UNE PF EFFICACE PAR LA COMMUNICATION DE COUPLE}

Le Projet de survie maternelle et infantile (MCSP) a mis en œuvre trois approches de communication de couple sur la PF dans le district de Kloto au Togo. Breakthrough RESEARCH et l'Institut de santé reproductive ont évalué deux de ces approches : les conseils à domicile et les discussions de groupe. L'objectif était d'évaluer les avantages et la faisabilité de ces deux approches pour renforcer l'acceptation et l'adhésion à la PF, et de déterminer quels éléments de chacune étaient les plus adaptés en vue d'un déploiement à plus grande échelle.

L'étude de cas qui en résulte examine de quelle manière les approches de communication de couple influencent les parcours et la prise de décisions associés aux comportements en matière de PF/SR des couples participants. De plus, elle décrit les expériences et les comportements des prestataires de services et des agents de santé communautaires (ASC) dans la conduite des activités de communication de couple. L'étude de cas explore également les changements de comportement éventuels des couples, ainsi que des prestataires et des ASC.

\footnotetext{
Pour de plus amples informations. Engaging Men for Effective Family Planning through Couple Communication: An Assessment of Two MCSP Couple Communication Approaches in Togo, https:// breakthroughactionandresearch.org/wp-content/ uploads/2019/09/Engaging-men-for-effective-FP-Togo.pdf.
} 


\section{MIEUX MESURER L'ÉTENDUE DES PROGRAMMES CSC EN PF/SR EN AFRIQUE DE L'OUEST FRANCOPHONE}

Breakthrough RESEARCH a procédé à une cartographie des investissements, des approches techniques et des indicateurs en matière de PF afin d'identifier les insuffisances des indicateurs et de formuler des recommandations destinées aux gouvernements, donateurs, chercheurs et agents de mise en œuvre, en vue de mieux évaluer les activités relatives au CSC dans le paysage programmatique élargie de la PF. Les chercheurs ont réalisé des entretiens structurés avec les parties prenantes en PF dans chaque pays et recueilli des documents, notamment des descriptions de programmes, des plans de suivi-évaluation et d'apprentissage et des fiches de référence d'indicateurs. L'équipe a analysé ces données en créant une matrice à partir de 1508 indicateurs provenant de 55 parties prenantes/projets. La matrice a servi de base aux cartes répertoriant les zones sensibles par type d'indicateur et de niveau socio-écologique. Des cartes indiquant les zones sensibles ont été initialement créées pour tous les indicateurs, puis pour les indicateurs spécifiques au CSC.

Plusieurs recommandations sur le suivi et l'évaluation du CSC ont émergé de ce travail et pourraient être appliquées aux programmes en cours et prévus sur la PF et le CSC en Afrique de l'Ouest francophone. À titre d'exemple, les conclusions du rapport ont donné lieu à des recommandations encourageant les donateurs à coordonner leurs efforts avec les partenaires au niveau national et régional afin de faciliter la standardisation des indicateurs du CSC et de garantir le suivi systématique des mesures liées au CSC. De plus, le rapport encourageait l'utilisation des données en adoptant des approches traduisant leur complexité dans des tableaux de bord, des cartes de données et des visualisations devant aider à communiquer les conclusions à des publics non-initiés.
Pour de plus amples informations. Strengthening Social and Behavior Change Monitoring and Evaluation for Family Planning in Francophone West Africa: Technical Report, http://breakthroughactionandresearch.org/wp-content/ uploads/2020/04/WABA-FP-IndicatorMapping-Report.pdf; Renforcement du suivi et de l'évaluation des changements sociaux et comportementaux pour le planning familial en Afrique de l'Ouest francophone, http://breakthroughactionandresearch.org/wpcontent/uploads/2020/09/BR_IndMap_WABA_Brief_Fr.pdf

\section{UTILISATION DE LA RECHERCHE ET GESTION DES CONNAISSANCES}

Breakthrough RESEARCH et son partenaire Population Reference Bureau veillent à ce que les connaissances découlant des activités en Afrique de l'Ouest francophone soient communiquées aux bons publics dans des formats adéquats pour informer les décideurs et encourager l'acceptation et l'efficacité des programmes sur le CSC dans la région. Breakthrough RESEARCH collabore étroitement avec Breakthrough ACTION et d'autres projets pour assurer la fluidité des flux d'informations dans les deux sens et veiller à ce que les résultats soient organisés, transmis et appliqués de manière à en optimiser la portée et l'impact.

En tant que projet de recherche, Breakthrough RESEARCH publie ses conclusions dans des revues académiques et ses résultats dans des exposés programmatiques plus digestes, des blogs, des webinaires et des diaporamas ciblant les donateurs, les agents de mise en œuvre et d'autres parties prenantes.

Les conclusions des activités de Breakthrough RESEARCH en Afrique de l'Ouest francophone ont été présentées lors de rencontres régionales, notamment à la Réunion annuelle du Partenariat de Ouagadougou, et seront communiquées visuellement ou en présentiel dans le monde, notamment dans des webinaires, des discussions sur Springboard pour le CSC, des blogs, une présentation des points de vue de Breakthrough ACTION et d'autres événements conjoints de Breakthrough RESEARCH et Breakthrough ACTION.

\section{Breakthrough RESEARCH}

Cette activité est mise en œuvre dans le cadre de Breakıthrough RESEARCH. Breakthrough RESEARCH accélère le CSC en effectuant une recherche de pointe et en valorisant des solutions factuelles en vue d'améliorer les programmes de santé et de développement dans le monde. Breakthrough RESEARCH est un consortium dirigé par le Population Council, en partenariat avec Avenir Health, ideas42, I'Institut de santé reproductive de Georgetown University, le Population Reference Bureau et Tulane University.

\section{Remerciements}

Breakthrough RESEARCH est rendu possible grâce au soutien généreux du peuple américain à travers l'Agence américaine pour le développement international (USAID) en vertu des termes de l'accord de coopération $n^{\circ}$ AID-OAA-A-17-00018. Le contenu de ce document relève de la responsabilité exclusive de Breakthrough RESEARCH et du Population Council et ne reflète pas nécessairement les positions de I'USAID ou du gouvernement des États-Unis.

\section{Breakthrough RESEARCH}

Population Council

4301 Connecticut Avenue, NW

Suite 280

Washington, DC 20008

Tél : +1 2022379400

breakthroughactionandresearch.org

\section{Crédit photographique}

(c) U.Ozel.Images / Getty Images 